\title{
CSG: Towards a comprehensive model of growth
}

\author{
Deniz Ünal and Begüm Çı̆̆şar \\ Department of Statistics, Faculty of Arts and Sciences, Çukurova University, Adana, Turkey
}

Received: 13 June 2021, Accepted: 16 June 2021

Published online: 12 July 2021.

\begin{abstract}
The main purpose of this study is to propose a better model than Sloboda and Gompertz models. So, a comprehensive growth model (CSG) is defined by combining Sloboda and Gompertz models, which are well known in literature and properties of the CSG model are given. In order to demonstrate the performance of the proposed model in modeling growth and to compare the performance of the new model with Gompertz and Sloboda models, an application was made on the real data set and the results were presented in table and graphics.
\end{abstract}

Keywords: Curve Fitting, Gompertz Growth Model, Sigmoid Functions, Sloboda Growth Model.

\section{Introduction}

Sigmoid functions are used in life and social sciences, physics and engineering to represent the growth. Sloboda (Sloboda, 1971) and Gompertz (Gompertz, 1825) growth models having sigmoidal structure represent the growth processes, by using first order differential equations. For example, population dynamics, artificial neural networks, signal and image processing, feeding techniques, financing and insurance are some of these fields (Kyurkchiev and Markov, 2015).

There are lots of studies using sigmoidal curves to model the growth and also to compare these functions about prediction accuracy. For example, seven sigmoid growth curve families were examined to determine the most suitable for pinus radiata according to age data. As a result, it has been shown that the three-parameter Chapman-Richards model and the four-parameter Levakovic and Sloboda models are superior to the Hossfeld, Schumacher, Weibull and Gompertz models (Lee, 2000). Gangying and Weitang (1996), defined the Sloboda growth model and they implemented its application on Chinese fir plantation and they stated that this model has satisfactory application effects. Another sigmoidal mortality function was given by Gompertz (1825). After a short time, the first development of Gompertz's law was given by Makeham in 1889 by modifications. After many years, Golubev (2009) proposed a model by using Makeham's model and Gompertz model.

In many of the above-mentioned studies, it is seen that MATLAB program and the least square curve fit (lsqrcurvefit) function are frequently used in cases such as solving differential equations and parameter estimation. For example; Benzekry et al. (2014) used the lsqrcurvefit function while applying exponential, exponential-linear, power law, Gompertz, Logistic, Generalized Logistic, von Bertalanffy and dynamic carrying capacity models to tumor growth. While comparing these models, they used Root Mean Square Error (RMSE) and Akaike Information (AIC) criteria. Windarto et al. (2018) presented a new model and compared Logistic, Richards and Gompertz growth models with $R^{2}$, Mean Absolute Percentage Error (MAPE) and Normalized Residual Sum of Squares (NRSS) metrics. 
In this study, we defined a unified growth model by integrating Gompertz and Sloboda functions. In the first part of the study Sloboda and Gompertz models are introduced. Also, the data set and the statistical criteria to be used in the study are introduced. In the second part, how to get the combined form using Sloboda and Gompertz sigmoidal functions, is given. Also, in the subsections of this part we present some properties of this combined form. Finally, a numerical

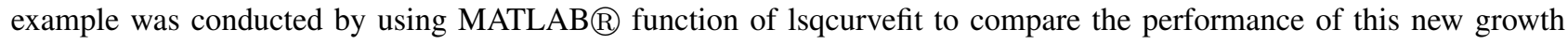
model defined by Sloboda and Gompertz functions.

\section{Materials and methods}

Sigmoidal Functions: For $S(x):[a, b] \rightarrow \mathbb{R}$ is a real valued function and $z \in \mathbb{R}$ is any parameter, if the function $S(x)$ becomes convex (or concave) for $x \leq z \in[a, b]$ and concave (or convex) for $x \geq z \in[a, b]$ these functions are called Sigmoidal functions.

Gompertz Function: General form of the Gompertz function can be given as follows

$$
y(t)=y_{\infty} . e^{-\alpha \cdot e^{-\beta t}}, t>0
$$

where $y(t)$ is current size of $\mathrm{y}$ at time $\mathrm{t}, y_{\infty}=\lim _{t \rightarrow \infty} y(t)$ is an asymptote, $\alpha$ is a shape parameter, $\beta$ is a scale parameter. Shape parameter determines the general shape of the curve. Scale parameter determines the scale of the statistical distribution and we know that the smaller the scale parameter gives the more concentrated the distribution.

Sloboda Function: The general form of the Sloboda function is

$$
y(t)=y_{\infty} \cdot e^{-\delta \cdot e^{\frac{b_{1}}{b_{2-1}} t^{1-b_{2}}}}
$$

where $\lim _{t \rightarrow \infty} y(t)=y_{\infty}$ and called as asymptotic limit of the number of total case or number of total deaths. Another presentation of this model is: $y(t)=y_{\infty} . e^{-\delta . e^{-m . t} \gamma}$ where $m=\frac{-b_{1}}{b_{2-1}}$ term is the percentage of the number of total cases in the number of total deaths. $\gamma=1-b_{2}$ term is the changing point for the curve's increasing to decreasing structure. The $\delta$ term is the percentage of the maximum increase rate in the highest number of cases or deaths.

\section{Construction of Combined Sloboda Gompertz Growth Model}

In this section, we establish the Combined Sloboda Gompertz (CSG) model and give some properties of this model.

Definition. For the combined model, let us define the instant rate of change in $y(t)$ at time $t$ as,

$$
\frac{\frac{d y(t)}{d t}}{y(t)}=\beta \cdot \ln \left(\frac{y_{\infty}}{y_{t}}\right)+(1-k) \cdot b_{1} \cdot t^{-b_{2}} \ln \left(\frac{y_{\infty}}{y_{t}}\right)
$$

Here the parameters are defined as given in Eq. 1 and Eq. 2 . The $k$ parameter is any real number in $0 \leq k \leq 1$. Performing some manipulations in Eq. 3 and taking integration

$$
\ln \left[\ln \left(\frac{y(t)}{y_{\infty}}\right)\right]=-\beta t-(1-k) \cdot b_{1} \cdot \frac{t^{1-b_{2}}}{1-b_{2}}+c
$$


can be obtained for arbitrary positive constant c value. Simplifying by exponential notation,

$$
\ln \left(\frac{y(t)}{y_{\infty}}\right)=e^{-\beta t-(1-k) \cdot b_{1} \cdot \frac{t^{1-b_{2}}}{1-b_{2}}+c}=e^{-\beta t} e^{-(1-k) \cdot b_{1} \cdot \frac{t^{1-b_{2}}}{1-b_{2}}} e^{c}
$$

is found. Without loss of generality let us take $e^{c}=\left(-c_{1}\right) .\left(-c_{2}\right)$ where $c_{1}$ and $c_{2}$ are arbitrary positive constants. By using the properties of logarithm, it can be seen that, the exponential parts on the right-hand side of the following equation are similar to Gompertz type (given in Equation 1) and Sloboda type (given in Equation 2) respectively:

$$
y(t)=y_{\infty} \cdot \exp \left(-c_{1} \cdot e^{-\beta t}\right) \exp \left(-c_{2} \cdot e^{-(1-k) \cdot b_{1} \cdot \frac{t^{1-b_{2}}}{1-b_{2}}}\right)
$$

Reducing this expression, for $\gamma=1-b_{2}, m=\frac{-b_{1}}{b_{2-1}}$ or $b_{1}=m \gamma$ and $b_{2}=1-\gamma$, the living size of CSG is given as follows

$$
y(t)=y_{\infty} \cdot \exp \left(-c_{1} \cdot e^{-\beta t}\right) \exp \left(-c_{2} \cdot e^{-m(1-k) \cdot t^{\gamma}}\right) .
$$

By arranging this equation, the living/population size of CSG model is

$$
y(t)=y_{\infty} \cdot e^{-c_{1} \cdot e^{-\beta t}} e^{-c_{2} \cdot e^{-m \cdot(1-k) \cdot t^{\gamma}}}
$$

\subsection{Properties of CSG growth model}

The fundamental characteristics of CSG expression is given in this section.

Initial Value: Since $y(t)$ is current size of $\mathrm{y}$ at time $\mathrm{t}$. Then for $\mathrm{t}=0$, the initial value for $y$ can be reached as follows:

$$
y_{0}=y(0)=y_{\infty} . e^{-c_{1}-c_{2}}
$$

This initial behavior gives the intercept on y axis for the growth curve.

Remark: Using Eq. 5 we can reach a restriction for $c_{1}$ and $c_{2}$ as, $c_{1}+c_{2}=\ln \left(\frac{y_{\infty}}{y_{0}}\right)$.

Upper Bound/Limit for Growth: Upper bound is the carrying capacity of a specific environment. In other words, this value is the horizontal asymptote for the growth model. It can be determined as $t$ tends to infinity:

$$
y_{\infty}=\lim _{t \rightarrow \infty} y(t)=\lim _{t \rightarrow \infty}\left(y_{\infty} \cdot e^{-c_{1} \cdot e^{-\beta t}} \cdot e^{-c_{2} \cdot e^{-m \cdot(1-k) \cdot t^{\gamma}}}\right) .
$$

This value gives the upper limit to growth parameter for CSG model.

Growth Rate: We can reach the growth rate by taking the first derivative of the combined growth form given in Equation 4.

$$
\frac{d y}{d t}=y^{\prime}(t)=y_{\infty} e^{-c_{1} \cdot e^{-\beta t}} e^{-c_{2} \cdot e^{-m \cdot(1-k) \cdot t^{\gamma}}}\left(c_{1} \beta e^{-\beta t}+m(1-k) c_{2} \gamma t^{\gamma-1} e^{-m \cdot(1-k) \cdot t^{\gamma}}\right)
$$

This gives the mathematical expression for the growth.

Remark: Initial behavior for the rate of growth is defined as follows:

$$
y^{\prime}(0)=y_{\infty} \cdot c_{1} \cdot \beta e^{-c_{1}-c_{2}}
$$


Critical Points: The values of $\mathrm{t}$ where $y^{\prime}(t)=0$ or where the function is not differentiable give the critical values. For the CSG function, $\mathrm{t}$ values that satisfy the following equation should give the critical points

$$
\frac{e^{m \cdot(1-k) \cdot t^{\gamma}-\beta t}}{t^{\gamma-1}}=\frac{m(k-1) c_{2} \gamma}{c_{1}}
$$

\section{Data Analysis}

In this section, to see the fitting performance of the CSG model and to make comparisons with Gompertz and Sloboda models, graphs and RMSE and MAPE values of these models are given by using Afghan Pikas dataset. This data set was recorded by Puget and Gouarderes (1974) and studied by Sandland and McGilchrist (1979) and consists of the weights of pregnant Afghan Pikas recorded in 14 simultaneous times.
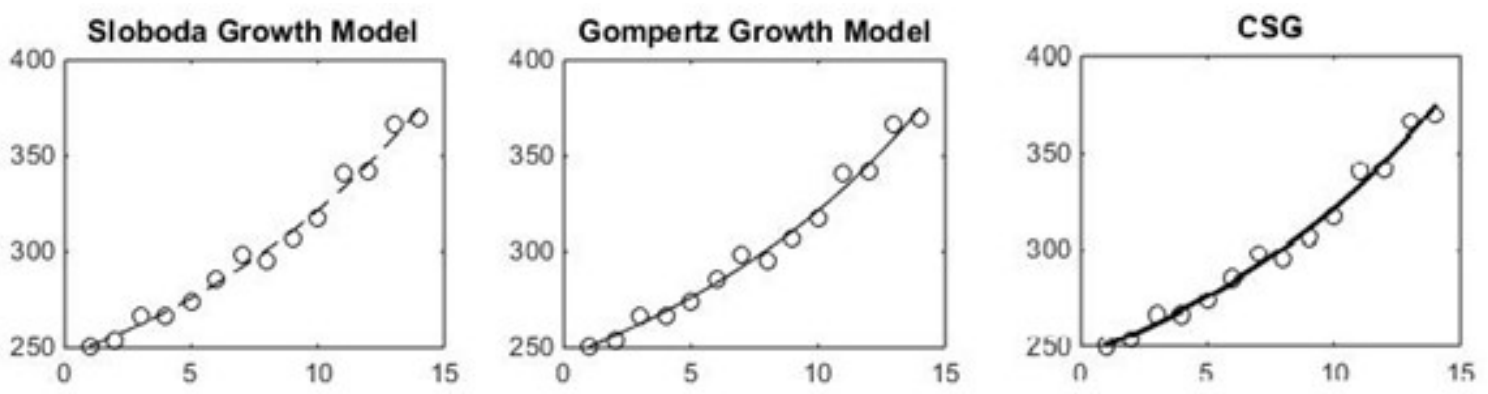

Fig. 1: Fitting the Afghan Pikas data with Sloboda, Gompertz and CSG models

Figure 1 shows the fitting of the data set to the models. So, it can be said that all models fit the data set quite well. In addition to this information, RMSE and MAPE criteria were used to compare models and determine the goodness of fit of the models. Calculated RMSE and MAPE metrics are given in Table 1.

\begin{tabular}{|l|l|l|}
\hline Model & RMSE & MAPE \\
\hline Gompertz & 4.5765 & 1.2969 \\
\hline Sloboda & 4.5762 & 1.2827 \\
\hline CSG Model & 4.5756 & 1.2821 \\
\hline
\end{tabular}

Table 1: RMSE and MAPE values of the models.

According to Table 1, it is observed that the defined model has a better performance since the RMSE and MAPE values are lower than the other models. This means that applying the CSG model in datasets where Gompertz or Sloboda models can be applied gives more accurate results.

\section{Conclusion}

Thanks to the studies on growth modeling and the obtained growth models, it is possible to predict the growth behavior of living things, the increase and decrease dynamics of the population of a certain species or the economic growth. In this study, a new one has been added to the growth models that can find a wide application area and make important 
predictions about the future. In the study, in which the theoretical form and important features of this new model, called CSG, were introduced, numerical analyzes were also made.

In the numerical part, using MATLAB $囚, \mathrm{CSG}$, Gompertz and Sloboda growth functions were applied to the Afghan Pikas (Puget and Gouarderes, 1974; Sandland and McGilchrist, 1979) data set (Figure 1). Later, these three models were compared according to RMSE and MAPE criteria (Table 1). As a result of the comparisons, it is seen that the CSG growth model fits the dataset better than Sloboda and Gompertz models in terms of all criteria.

This shows that the CSG model performs better modeling than Gompertz and Sloboda functions. In the light of these results, in future studies where Gompertz or Sloboda models can be applied, applying the obtained CSG model instead of applying these models alone will provide better results.

\section{Competing interests}

The authors declare that they have no competing interests.

\section{Authors' contributions}

All authors have contributed to all parts of the article. All authors read and approved the final manuscript.

\section{Acknowledgement}

This study was supported by Çukurova University Scientific Research Projects Coordination Unit. Project Number: FDK2019-12338.

\section{References}

[1] Benzekry, S., Lamont, C., Beheshti, A., Tracz, A., \& Ebos, J. M. L. (2014). Classical Mathematical Models for Description and Prediction of Experimental Tumor.

[2] Gangying, H., Weitong, S. Sloboda height growth model and its application on Chinese fir plantation Forest Research. 1996 ;9(1):37-40.

[3] Golubev, A. (2009). How could the Gompertz-Makeham law evolve. Journal of theoretical Biology, 258(1), 1-17.

[4] Gompertz, B. (1825). XXIV. On the nature of the function expressive of the law of human mortality, and on a new mode of determining the value of life contingencies. In a letter to Francis Baily, Esq. FRS \&c. Philosophical transactions of the Royal Society of London, (115), 513-583.

[5] Kyurkchiev, N., \& Markov, S. (2015). Sigmoid functions: some approximation and modelling aspects. LAP LAMBERT Academic Publishing, Saarbrucken.

[6] Lee, A. (2000). The predictive ability of seven sigmoid curves used in modelling forestry growth: a thesis submitted to the Institute of Information Sciences and Technology in partial fulfilment of the requirements for the degree of Master of Applied Statistics at Massey University, February, 2000 (Doctoral dissertation, Massey University).

[7] Makeham, W. M. (1889). On the further development of Gompertz's law. Journal of the Institute of Actuaries (1886-1994), 28(2), 152-159.

[8] Puget, A. (1974). Weight Gain of The Afghan Pika (Ochotona Sufescens Rufescens) From Birth To 19 Weeks Of Age, And During Gestation.

[9] Sandland, R. L., \& McGilchrist, C. A. (1979). Stochastic growth curve analysis. Biometrics, 255-271. 
[10] Sloboda B (1971). For the representation of growth processes with the help of differential equations of the first order, notifications of the Baden-Wurttemberg forestry experimental and research institute, 32.

[11] Windarto, Bridani, Pwoti, U.D.(2018). A new modified logistic growth model for empirical use. Communication in Biomathematical Sciences, 1(2). 